\title{
An Indication of Anisotropy in Arrival Directions of Ultra-high-energy Cosmic Rays through Comparison to the Flux Pattern of Extragalactic Gamma-Ray Sources
}

A. Aab ${ }^{1}$, P. Abreu ${ }^{2}$, M. Aglietta ${ }^{3,4}$, I. F. M. Albuquerque ${ }^{5}$, I. Allekotte ${ }^{6}$, A. Almela ${ }^{7,8}$, J. Alvarez Castillo ${ }^{9}$, J. Alvarez-Muñiz ${ }^{10}$, G. A. Anastasi ${ }^{11,12}$, L. Anchordoqui ${ }^{13}$, B. Andrada ${ }^{7}$, S. Andringa ${ }^{2}$, C. Aramo ${ }^{14}$, N. Arsene ${ }^{15}$, H. Asorey ${ }^{6,16}$, P. Assis ${ }^{2}$, G. Avila ${ }^{17,18}$, A. M. Badescu ${ }^{19}$, A. Balaceanu ${ }^{20}$, F. Barbato ${ }^{21}$, R. J. Barreira Luz ${ }^{2}$, J. J. Beatty ${ }^{22}$, K. H. Becker ${ }^{23}$, J. A. Bellido ${ }^{24}$, C. Berat ${ }^{25}$, M. E. Bertaina ${ }^{3,26}$, X. Bertou ${ }^{6}$, P. L. Biermann ${ }^{27}$, J. Biteau ${ }^{28}$, S. G. Blaess ${ }^{24}$, A. Blanco ${ }^{2}$, J. Blazek ${ }^{29}$, C. Bleve ${ }^{30,31}$, M. Boháčová ${ }^{29}$, C. Bonifazi ${ }^{32}$, N. Borodai ${ }^{33}$, A. M. Botti ${ }^{7,34}$, J. Brack ${ }^{35}$, I. Brancus ${ }^{20,98}$, T. Bretz ${ }^{36}$, A. Bridgeman ${ }^{37}$, F. L. Briechle ${ }^{36}$, P. Buchholz ${ }^{38}$, A. Bueno $^{39}$, S. Buitink ${ }^{1}$, M. Buscemi ${ }^{40,41}$, K. S. Caballero-Mora ${ }^{42}$, L. Caccianiga ${ }^{43}$, A. Cancio ${ }^{7,8}$, F. Canfora ${ }^{1}$, R. Caruso ${ }^{40,41}$,

A. Castellina ${ }^{3,4}$, F. Catalani ${ }^{44}$, G. Cataldi ${ }^{30}$, L. Cazon $^{2}$, A. G. Chavez ${ }^{45}$, J. A. Chinellato ${ }^{46}$, J. Chudoba ${ }^{29}$, R. W. Clay ${ }^{24}$,

A. C. Cobos Cerutti ${ }^{47}$, R. Colalillo ${ }^{14,21}$, A. Coleman $^{48}$, L. Collica ${ }^{3}$, M. R. Coluccia ${ }^{30,31}$, R. Conceição ${ }^{2}$, G. Consolati ${ }^{49,50}$,

F. Contreras ${ }^{17,18}$, M. J. Cooper ${ }^{24}$, S. Coutu ${ }^{48}$, C. E. Covault ${ }^{51}$, J. Cronin ${ }^{52,98}$, S. D’Amico ${ }^{30,53}$, B. Daniel ${ }^{46}$, S. Dasso ${ }^{54,55}$,

K. Daumiller ${ }^{34}$, B. R. Dawson ${ }^{24}$, R. M. de Almeida ${ }^{56}$, S. J. de Jong ${ }^{1,57}$, G. De Mauro ${ }^{1}$, J. R. T. de Mello Neto ${ }^{32,58}$, I. De Mitri ${ }^{30,31}$, J. de Oliveira ${ }^{56}$, V. de Souza $^{59}$, J. Debatin ${ }^{37}$, O. Deligny ${ }^{28}$, M. L. Díaz Castro ${ }^{46}$, F. Diogo ${ }^{2}$, C. Dobrigkeit ${ }^{46}$, J. C. D’Olivo ${ }^{9}$, Q. Dorosti ${ }^{38}$, R. C. dos Anjos ${ }^{60}$, M. T. Dova ${ }^{61}$, A. Dundovic ${ }^{62}$, J. Ebr ${ }^{29}$, R. Engel ${ }^{34}$, M. Erdmann ${ }^{36}$, M. Erfani ${ }^{38}$, C. O. Escobar ${ }^{63}$, J. Espadanal ${ }^{2}$, A. Etchegoyen ${ }^{7,8}$, H. Falcke ${ }^{1,57,64}$, J. Farmer ${ }^{52}$, G. Farrar ${ }^{65}$, A. C. Fauth ${ }^{46}$, N. Fazzini ${ }^{63}$, F. Fenu ${ }^{3,26}$, B. Fick ${ }^{66}$, J. M. Figueira ${ }^{7}$, A. Filipčič ${ }^{67,68}$, M. M. Freire ${ }^{69}$, T. Fujii ${ }^{52}$, A. Fuster ${ }^{7,8}$, R. Gaïor ${ }^{70}$, B. García ${ }^{47}$, F. Gaté ${ }^{71}$, H. Gemmeke ${ }^{72}$, A. Gherghel-Lascu ${ }^{20}$, P. L. Ghia ${ }^{28}$, U. Giaccari ${ }^{32}$, M. Giammarchi ${ }^{49}$, M. Giller ${ }^{73}$, D. Głas ${ }^{74}$, C. Glaser ${ }^{36}$, G. Golup ${ }^{6}$,

M. Gómez Berisso ${ }^{6}$, P. F. Gómez Vitale ${ }^{17,18}$, N. González ${ }^{7,34}$, A. Gorgi ${ }^{3,4}$, A. F. Grillo ${ }^{12,98}$, T. D. Grubb ${ }^{24}$, F. Guarino ${ }^{14,21}$, G. P. Guedes ${ }^{75}$, R. Halliday ${ }^{51}$, M. R. Hampel ${ }^{7}$, P. Hansen ${ }^{61}$, D. Harari ${ }^{6}$, T. A. Harrison ${ }^{24}$, A. Haungs ${ }^{34}$, T. Hebbeker ${ }^{36}$, D. Heck ${ }^{34}$, P. Heimann ${ }^{38}$, A. E. Herve ${ }^{37}$, G. C. Hill ${ }^{24}$, C. Hojvat ${ }^{63}$, E. Holt ${ }^{7,34}$, P. Homola ${ }^{33}$, J. R. Hörandel ${ }^{1,57}$, P. Horvath ${ }^{76}$, M. Hrabovský ${ }^{76}$, T. Huege ${ }^{34}$, J. Hulsman ${ }^{7,34}$, A. Insolia ${ }^{40,41}$, P. G. Isar ${ }^{15}$, I. Jandt ${ }^{23}$, J. A. Johnsen ${ }^{77}$, M. Josebachuili ${ }^{7}$, J. Jurysek ${ }^{29}$, A. Kääpä ${ }^{23}$, O. Kambeitz ${ }^{37}$, K. H. Kampert ${ }^{23}$, B. Keilhauer ${ }^{34}$, N. Kemmerich ${ }^{5}$, E. Kemp ${ }^{46}$, J. Kemp ${ }^{36}$, R. M. Kieckhafer ${ }^{66}$, H. O. Klages ${ }^{34}$, M. Kleifges ${ }^{72}$, J. Kleinfeller ${ }^{17}$, R. Krause ${ }^{36}$, N. Krohm ${ }^{23}$, D. Kuempel ${ }^{23}$, G. Kukec Mezek ${ }^{67}$, N. Kunka ${ }^{72}$, A. Kuotb Awad ${ }^{37}$, B. L. Lago ${ }^{78}$, D. LaHurd ${ }^{51}$, R. G. Lang ${ }^{59}$, M. Lauscher ${ }^{36}$, R. Legumina ${ }^{73}$, M. A. Leigui de Oliveira ${ }^{79}$, A. Letessier-Selvon ${ }^{70}$, I. Lhenry-Yvon ${ }^{28}$, K. Link ${ }^{37}$, D. Lo Presti ${ }^{41}$, L. Lopes ${ }^{2}$, R. López ${ }^{80}$, A. López Casado ${ }^{10}$, R. Lorek ${ }^{51}$, Q. Luce ${ }^{28}$, A. Lucero ${ }^{7}$, M. Malacari ${ }^{52}$, M. Mallamaci ${ }^{49,43}$, D. Mandat ${ }^{29}$, P. Mantsch ${ }^{63}$, A. G. Mariazzi ${ }^{61}$, I. C. Mariş ${ }^{81}$, G. Marsella ${ }^{30,31}$, D. Martello ${ }^{30,31}$,

H. Martinez ${ }^{82}$, O. Martínez Bravo ${ }^{80}$, J. J. Masías Meza ${ }^{54}$, H. J. Mathes ${ }^{34}$, S. Mathys ${ }^{23}$, J. Matthews $^{83}$, G. Matthiae ${ }^{84,85}$,

E. Mayotte $^{23}$, P. O. Mazur ${ }^{63}$, C. Medina ${ }^{77}$, G. Medina-Tanco ${ }^{9}$, D. Melo ${ }^{7}$, A. Menshikov ${ }^{72}$, K.-D. Merenda ${ }^{77}$, S. Michal ${ }^{76}$, M. I. Micheletti ${ }^{69}$, L. Middendorf ${ }^{36}$, L. Miramonti ${ }^{49,43}$, B. Mitrica ${ }^{20}$, D. Mockler ${ }^{37}$, S. Mollerach ${ }^{6}$, F. Montanet ${ }^{25}$, C. Morello ${ }^{3,4}$, G. Morlino ${ }^{11,12}$, M. Mostafá ${ }^{48}$, A. L. Müller ${ }^{7,34}$, G. Müller ${ }^{36}$, M. A. Muller ${ }^{46,86}$, S. Müller ${ }^{7,37}$, R. Mussa ${ }^{3}$, I. Naranjo ${ }^{6}$, L. Nellen ${ }^{9}$, P. H. Nguyen ${ }^{24}$, M. Niculescu-Oglinzanu ${ }^{20}$, M. Niechciol ${ }^{38}$, L. Niemietz ${ }^{23}$, T. Niggemann ${ }^{36}$, D. Nitz ${ }^{66}$, D. Nosek ${ }^{87}$, V. Novotny ${ }^{87}$, L. Nožka ${ }^{76}$, L. A. Núñez ${ }^{16}$, F. Oikonomou ${ }^{48}$, A. Olinto ${ }^{52}$, M. Palatka ${ }^{29}$, J. Pallotta ${ }^{88}$, P. Papenbreer ${ }^{23}$, G. Parente ${ }^{10}$, A. Parra ${ }^{80}$,

T. Paul ${ }^{13}$, M. Pech ${ }^{29}$, F. Pedreira ${ }^{10}$, J. Pȩkala ${ }^{33}$, R. Pelayo ${ }^{89}$, J. Peña-Rodriguez ${ }^{16}$, L. A. S. Pereira ${ }^{46}$, M. Perlin ${ }^{7}$, L. Perrone $^{30,31}$, C. Peters ${ }^{36}$, S. Petrera ${ }^{11,12}$, J. Phuntsok ${ }^{48}$, T. Pierog ${ }^{34}$, M. Pimenta ${ }^{2}$, V. Pirronello ${ }^{40,41}$, M. Platino ${ }^{7}$, M. Plum ${ }^{36}$, J. Poh ${ }^{52}$, C. Porowski ${ }^{33}$, R. R. Prado ${ }^{59}$, P. Privitera ${ }^{52}$, M. Prouza ${ }^{29}$, E. J. Quel ${ }^{88}$, S. Querchfeld ${ }^{23}$, S. Quinn ${ }^{51}$, R. Ramos-Pollan ${ }^{16}$, J. Rautenberg ${ }^{23}$, D. Ravignani ${ }^{7}$, J. Ridky ${ }^{29}$, F. Riehn ${ }^{2}$, M. Risse ${ }^{38}$, P. Ristori ${ }^{88}$, V. Rizi ${ }^{12,90}$, W. Rodrigues de Carvalho ${ }^{5}$, G. Rodriguez Fernandez ${ }^{84,85}$, J. Rodriguez Rojo ${ }^{17}$, M. J. Roncoroni ${ }^{7}$, M. Roth ${ }^{34}$, E. Roulet ${ }^{6}$, A. C. Rovero ${ }^{55}$, P. Ruehl ${ }^{38}$, S. J. Saffi ${ }^{24}$, A. Saftoiu ${ }^{20}$, F. Salamida ${ }^{12,90}$, H. Salazar ${ }^{80}$, A. Saleh ${ }^{67}$, G. Salina ${ }^{84}$, F. Sánchez ${ }^{7}$, P. Sanchez-Lucas ${ }^{39}$, E. M. Santos ${ }^{5}$, E. Santos ${ }^{7}$, F. Sarazin ${ }^{77}$, R. Sarmento ${ }^{2}$, C. Sarmiento-Cano ${ }^{7}$, R. Sato ${ }^{17}$, M. Schauer ${ }^{23}$, V. Scherini ${ }^{30}$, H. Schieler ${ }^{34}$, M. Schimp ${ }^{23}$, D. Schmidt ${ }^{7,34}$, O. Scholten ${ }^{91,92}$, P. Schovánek ${ }^{29}$, F. G. Schröder ${ }^{34}$, S. Schröder ${ }^{23}$, A. Schulz ${ }^{37}$, J. Schumacher ${ }^{36}$, S. J. Sciutto ${ }^{61}$, A. Segreto ${ }^{40,93}$, A. Shadkam ${ }^{83}$, R. C. Shellard ${ }^{94}$, G. Sigl ${ }^{62}$, G. Silli ${ }^{7,34}$, R. Šmída ${ }^{34}$, G. R. Snow ${ }^{95}$, P. Sommers ${ }^{48}$, S. Sonntag ${ }^{38}$, J. F. Soriano ${ }^{13}$, R. Squartini ${ }^{17}$, D. Stanca ${ }^{20}$, S. Stanic ${ }^{67}$, J. Stasielak ${ }^{33}$, P. Stassi ${ }^{25}$, M. Stolpovskiy ${ }^{25}$, F. Strafella ${ }^{30,31}$, A. Streich ${ }^{37}$,

F. Suarez ${ }^{7,8}$, M. Suarez Durán ${ }^{16}$, T. Sudholz ${ }^{24}$, T. Suomijärvi ${ }^{28}$, A. D. Supanitsky ${ }^{55}$, J. Supík ${ }^{76}$, J. Swain ${ }^{96}$, Z. Szadkowski ${ }^{74}$,

A. Taboada $^{34}$, O. A. Taborda ${ }^{6}$, V. M. Theodoro ${ }^{46}$, C. Timmermans ${ }^{1,57}$, C. J. Todero Peixoto ${ }^{44}$, L. Tomankova ${ }^{34}$, B. Tomé2 ${ }^{2}$

G. Torralba Elipe ${ }^{10}$, P. Travnicek ${ }^{29}$, M. Trini ${ }^{67}$, R. Ulrich ${ }^{34}$, M. Unger $^{34}$, M. Urban ${ }^{36}$, J. F. Valdés Galicia ${ }^{9}$, I. Valiño ${ }^{10}$,

L. Valore ${ }^{14,21}$, G. van Aar ${ }^{1}$, P. van Bodegom ${ }^{24}$, A. M. van den Berg ${ }^{91}$, A. van Vliet ${ }^{1}$, E. Varela ${ }^{80}$, B. Vargas Cárdenas ${ }^{9}$,

R. A. Vázquez ${ }^{10}$, D. Veberič ${ }^{34}$, C. Ventura ${ }^{58}$, I. D. Vergara Quispe ${ }^{61}$, V. Verzi ${ }^{84}$, J. Vicha ${ }^{29}$, L. Villaseñor ${ }^{45}$, S. Vorobiov ${ }^{67}$, H. Wahlberg ${ }^{61}$, O. Wainberg ${ }^{7,8}$, D. Walz ${ }^{36}$, A. A. Watson ${ }^{97}$, M. Weber ${ }^{72}$, A. Weindl ${ }^{34}$, M. Wiedeński ${ }^{74}$, L. Wiencke ${ }^{77}$, H. Wilczyński ${ }^{33}$, M. Wirtz ${ }^{36}$, D. Wittkowski ${ }^{23}$, B. Wundheiler ${ }^{7}$, L. Yang ${ }^{67}$, A. Yushkov ${ }^{7}$, E. Zas ${ }^{10}$, D. Zavrtanik ${ }^{67,68}$, M. Zavrtanik ${ }^{67,68}$, A. Zepeda ${ }^{82}$, B. Zimmermann ${ }^{72}$, M. Ziolkowski ${ }^{38}$, Z. Zong ${ }^{28}$, and F. Zuccarello ${ }^{40,41}$

(The Pierre Auger Collaboration) 
${ }^{1}$ IMAPP, Radboud University Nijmegen, Nijmegen, The Netherlands

${ }^{2}$ Laboratório de Instrumentação e Física Experimental de Partículas—LIP and Instituto Superior Técnico—IST, Universidade de Lisboa—UL, Lisboa, Portugal ${ }^{3}$ INFN, Sezione di Torino, Torino, Italy

${ }^{4}$ Osservatorio Astrofisico di Torino (INAF), Torino, Italy

${ }^{5}$ Universidade de São Paulo, Instituto de Física, São Paulo, SP, Brazil

${ }^{6}$ Centro Atómico Bariloche and Instituto Balseiro (CNEA-UNCuyo-CONICET), San Carlos de Bariloche, Argentina

7 Instituto de Tecnologías en Detección y Astropartículas (CNEA, CONICET, UNSAM), Buenos Aires, Argentina

${ }^{8}$ Universidad Tecnológica Nacional-Facultad Regional Buenos Aires, Buenos Aires, Argentina

Universidad Nacional Autónoma de México, México, D.F., México

${ }^{10}$ Universidad de Santiago de Compostela, Santiago de Compostela, Spain

${ }^{11}$ Gran Sasso Science Institute (INFN), L'Aquila, Italy

${ }^{12}$ INFN Laboratori Nazionali del Gran Sasso, Assergi (L'Aquila), Italy

${ }^{13}$ Department of Physics and Astronomy, Lehman College, City University of New York, Bronx, NY, USA

${ }^{14}$ INFN, Sezione di Napoli, Napoli, Italy

${ }^{15}$ Institute of Space Science, Bucharest-Magurele, Romania

${ }^{16}$ Universidad Industrial de Santander, Bucaramanga, Colombia

${ }^{17}$ Observatorio Pierre Auger, Malargüe, Argentina

${ }^{18}$ Observatorio Pierre Auger and Comisión Nacional de Energía Atómica, Malargüe, Argentina

${ }^{19}$ University Politehnica of Bucharest, Bucharest, Romania

20 "Horia Hulubei" National Institute for Physics and Nuclear Engineering, Bucharest-Magurele, Romania

${ }^{21}$ Università di Napoli "Federico II," Dipartimento di Fisica "Ettore Pancini," Napoli, Italy ${ }^{22}$ Ohio State University, Columbus, OH, USA

${ }^{23}$ Bergische Universität Wuppertal, Department of Physics, Wuppertal, Germany

${ }^{24}$ University of Adelaide, Adelaide, S.A., Australia

${ }^{25}$ Laboratoire de Physique Subatomique et de Cosmologie (LPSC), Université Grenoble-Alpes, CNRS/IN2P3, Grenoble, France

26 Università Torino, Dipartimento di Fisica, Torino, Italy

${ }^{27}$ Max-Planck-Institut für Radioastronomie, Bonn, Germany

${ }^{28}$ Institut de Physique Nucléaire d'Orsay (IPNO), Université Paris-Sud, Univ. Paris/Saclay, CNRS-IN2P3, Orsay, France

${ }^{29}$ Institute of Physics of the Czech Academy of Sciences, Prague, Czech Republic

${ }^{30}$ INFN, Sezione di Lecce, Lecce, Italy

${ }^{31}$ Università del Salento, Dipartimento di Matematica e Fisica "E. De Giorgi," Lecce, Italy

${ }^{32}$ Universidade Federal do Rio de Janeiro, Instituto de Física, Rio de Janeiro, RJ, Brazil

33 Institute of Nuclear Physics PAN, Krakow, Poland

${ }^{34}$ Karlsruhe Institute of Technology, Institut für Kernphysik, Karlsruhe, Germany

${ }^{35}$ Colorado State University, Fort Collins, CO, USA

${ }^{36}$ RWTH Aachen University, III. Physikalisches Institut A, Aachen, Germany

${ }^{37}$ Karlsruhe Institute of Technology, Institut für Experimentelle Kernphysik (IEKP), Karlsruhe, Germany

${ }^{38}$ Universität Siegen, Fachbereich 7 Physik-Experimentelle Teilchenphysik, Siegen, Germany

${ }^{39}$ Universidad de Granada and C.A.F.P.E., Granada, Spain

${ }^{40}$ INFN, Sezione di Catania, Catania, Italy

${ }^{41}$ Università di Catania, Dipartimento di Fisica e Astronomia, Catania, Italy

42 Universidad Autónoma de Chiapas, Tuxtla Gutiérrez, Chiapas, México

${ }^{43}$ Università di Milano, Dipartimento di Fisica, Milano, Italy

${ }_{45}^{44}$ Universidade de São Paulo, Escola de Engenharia de Lorena, Lorena, SP, Brazil

${ }^{45}$ Universidad Michoacana de San Nicolás de Hidalgo, Morelia, Michoacán, México

${ }^{46}$ Universidade Estadual de Campinas, IFGW, Campinas, SP, Brazil

${ }^{47}$ Instituto de Tecnologías en Detección y Astropartículas (CNEA, CONICET, UNSAM), and Universidad Tecnológica Nacional—Facultad Regional Mendoza (CONICET/CNEA), Mendoza, Argentina

${ }^{48}$ Pennsylvania State University, University Park, PA, USA

${ }_{49}$ INFN, Sezione di Milano, Milano, Italy

${ }^{50}$ Politecnico di Milano, Dipartimento di Scienze e Tecnologie Aerospaziali, Milano, Italy

${ }^{51}$ Case Western Reserve University, Cleveland, OH, USA

${ }_{53}^{52}$ University of Chicago, Enrico Fermi Institute, Chicago, IL, USA

${ }^{53}$ Università del Salento, Dipartimento di Ingegneria, Lecce, Italy

${ }^{54}$ Departamento de Física and Departamento de Ciencias de la Atmósfera y los Océanos, FCEyN, Universidad de Buenos Aires and CONICET, Buenos Aires, Argentina

${ }^{55}$ Instituto de Astronomía y Física del Espacio (IAFE, CONICET-UBA), Buenos Aires, Argentina

${ }^{56}$ Universidade Federal Fluminense, EEIMVR, Volta Redonda, RJ, Brazil

${ }^{57}$ Nationaal Instituut voor Kernfysica en Hoge Energie Fysica (NIKHEF), Science Park, Amsterdam, The Netherlands

${ }^{58}$ Universidade Federal do Rio de Janeiro (UFRJ), Observatório do Valongo, Rio de Janeiro, RJ, Brazil

${ }^{59}$ Universidade de São Paulo, Instituto de Física de São Carlos, São Carlos, SP, Brazil

${ }^{60}$ Universidade Federal do Paraná, Setor Palotina, Palotina, Brazil

${ }_{62}^{61}$ IFLP, Universidad Nacional de La Plata and CONICET, La Plata, Argentina

${ }^{62}$ Universität Hamburg, II. Institut für Theoretische Physik, Hamburg, Germany

${ }^{63}$ Fermi National Accelerator Laboratory, Batavia, IL, USA

${ }^{64}$ Stichting Astronomisch Onderzoek in Nederland (ASTRON), Dwingeloo, The Netherlands

${ }^{65}$ New York University, New York, NY, USA

${ }^{66}$ Michigan Technological University, Houghton, MI, USA

${ }^{67}$ Center for Astrophysics and Cosmology (CAC), University of Nova Gorica, Nova Gorica, Slovenia

${ }^{68}$ Experimental Particle Physics Department, J. Stefan Institute, Ljubljana, Slovenia

${ }^{69}$ Instituto de Física de Rosario (IFIR)—CONICET/U.N.R. and Facultad de Ciencias Bioquímicas y Farmacéuticas U.N.R., Rosario, Argentina

${ }^{70}$ Laboratoire de Physique Nucléaire et de Hautes Energies (LPNHE), Universités Paris 6 et Paris 7, CNRS-IN2P3, Paris, France

${ }^{71}$ SUBATECH, École des Mines de Nantes, CNRS-IN2P3, Université de Nantes, France

${ }^{72}$ Karlsruhe Institute of Technology, Institut für Prozessdatenverarbeitung und Elektronik, Karlsruhe, Germany

${ }^{73}$ University of Łódź, Faculty of Astrophysics, Łódź, Poland

74 University of Łódź, Faculty of High-Energy Astrophysics, Łódź, Poland 


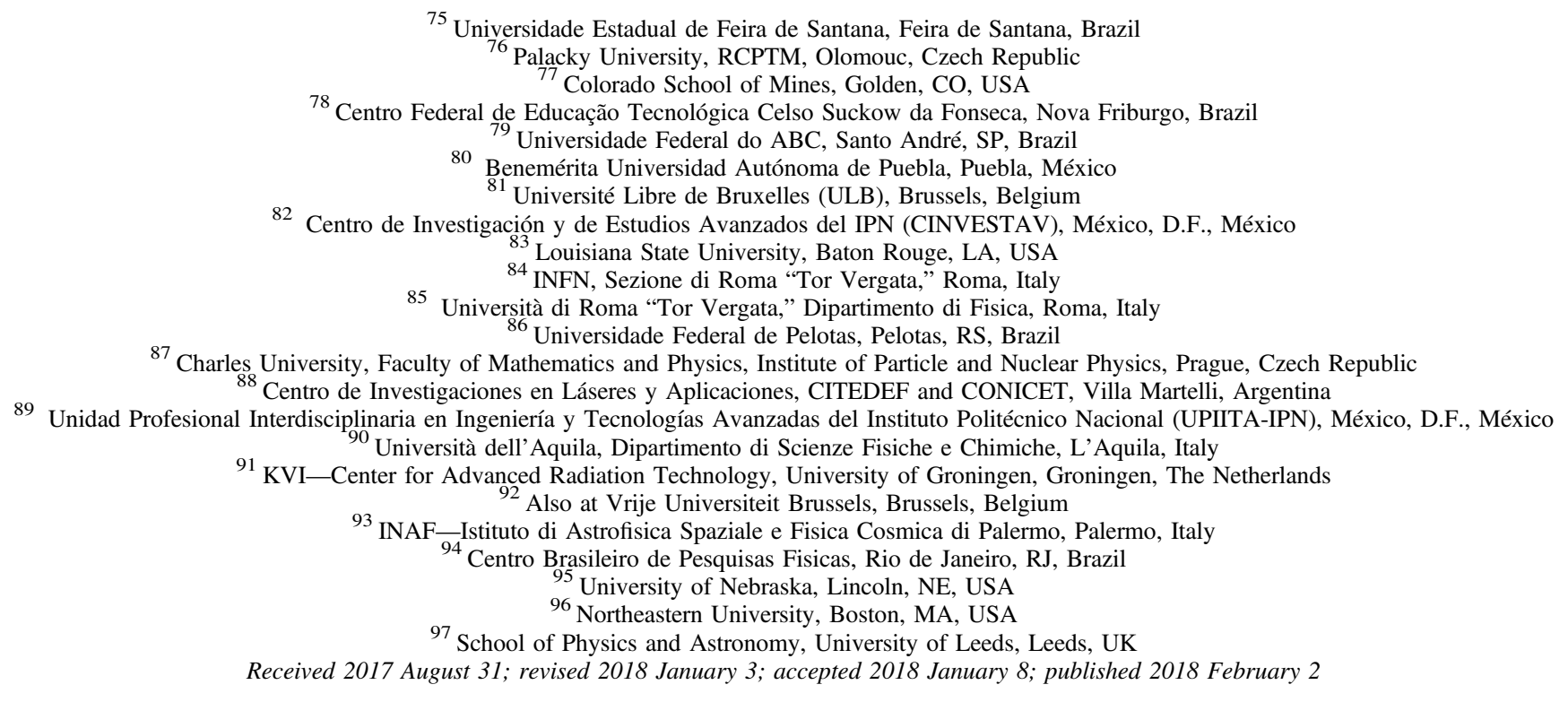

\begin{abstract}
A new analysis of the data set from the Pierre Auger Observatory provides evidence for anisotropy in the arrival directions of ultra-high-energy cosmic rays on an intermediate angular scale, which is indicative of excess arrivals from strong, nearby sources. The data consist of 5514 events above $20 \mathrm{EeV}$ with zenith angles up to $80^{\circ}$ recorded before 2017 April 30. Sky models have been created for two distinct populations of extragalactic gamma-ray emitters: active galactic nuclei from the second catalog of hard Fermi-LAT sources (2FHL) and starburst galaxies from a sample that was examined with Fermi-LAT. Flux-limited samples, which include all types of galaxies from the Swift-BAT and 2MASS surveys, have been investigated for comparison. The sky model of cosmic-ray density constructed using each catalog has two free parameters, the fraction of events correlating with astrophysical objects, and an angular scale characterizing the clustering of cosmic rays around extragalactic sources. A maximum-likelihood ratio test is used to evaluate the best values of these parameters and to quantify the strength of each model by contrast with isotropy. It is found that the starburst model fits the data better than the hypothesis of isotropy with a statistical significance of $4.0 \sigma$, the highest value of the test statistic being for energies above $39 \mathrm{EeV}$. The three alternative models are favored against isotropy with $2.7 \sigma-3.2 \sigma$ significance. The origin of the indicated deviation from isotropy is examined and prospects for more sensitive future studies are discussed.
\end{abstract}

Key words: astroparticle physics - cosmic rays - galaxies: active - galaxies: starburst - methods: data analysis

Supporting material: FITS files

\section{The Search for UHECR Anisotropies}

Identifying the sources of ultra-high-energy cosmic rays (UHECRs) has been a prime goal of particle astrophysics for decades. The challenge is considerable because the flux falls rapidly with increasing energy, and because UHECRs have a mixed mass composition (Aab et al. 2014a, 2016a), so some or all of them experience substantial magnetic deflections. Many scenarios have been proposed involving different populations of host galaxies. In this Letter, we investigate whether intermediate-scale ${ }^{99}$ anisotropies in UHECR arrival directions are associated with either or both of two prominent classes of extragalactic sources detected by Fermi-LAT-active galactic nuclei (AGNs) and starburst galaxies (SBGs)—using the gamma-ray luminosity or its surrogate (radio emission for SBGs) as a proxy for the relative luminosity of each source in UHECRs.

\footnotetext{
98 Deceased.

99 "Intermediate" hereafter denotes angular scales larger than the experimental resolution, $\sim 1^{\circ}$, and smaller than large-scale patterns, $\gtrsim 45^{\circ}$.
}

The rate of energy production of UHECRs determined from observations above $10^{18} \mathrm{eV}$ is close to $10^{45} \mathrm{erg} \mathrm{Mpc}^{-3} \mathrm{yr}^{-1}$ (Unger et al. 2015). Based on the Fermi-LAT survey, Dermer \& Razzaque (2010) argued that AGNs and SBGs match such rates in the gamma-ray band. Due to the low density of the detected SBGs and AGNs, and the attenuation of UHECR flux with increasing distance (GZK effect, Greisen 1966; Zatsepin \& Kuz'min 1966), a few objects would be expected to dominate the local flux, naturally producing an intermediatescale anisotropy if these sources contribute a sufficient fraction of the UHECR flux.

The AGN and SBG populations are well-motivated physically. AGNs are favored source candidates because their jets and radio lobes satisfy the Hillas criterion for shock acceleration (Hillas 1984). SBGs-being loci of intense star formation-potentially have increased rates of extreme events associated with the deaths of short-lived, massive stars, such as gamma-ray bursts, hypernovae, and magnetars (see, e.g., Biermann et al. 2016; Perley et al. 2016). Their winds have also been proposed as possible reacceleration sites (Anchordoqui et al. 1999). 
The analysis presented here is an advance in several ways. First, Fermi-LAT observations of gamma-rays from two extragalactic populations provide us with possible ansatzes for the relative UHECR fluxes from source candidates. That information makes the present analyses potentially more sensitive than previous studies based solely on the source direction. Second, thanks to our improved knowledge of the energydependent composition, we can now account more accurately for the relative attenuation of fluxes from distant sources. Third, thanks to the significant increase in exposure of the Pierre Auger Observatory with respect to previous analyses, the data can reveal more subtle patterns.

\section{UHECR Data Set}

UHECRs are detected at the Pierre Auger Observatory (Argentina, latitude 35.2 S, longitude 69.5 W; Aab et al. 2015c) through the extensive air showers they induce in the atmosphere. Air showers are detected on the ground with an array of 1600 water-Cherenkov detectors with a duty cycle of nearly $100 \%$. Twenty-four fluorescence telescopes map, during dark nights (duty cycle of $\sim 15 \%$ ), the longitudinal profile of each shower via the nitrogen fluorescence produced dominantly by the electromagnetic cascade. The combination of both techniques provides the array with an energy scale insensitive to primary mass assumptions and air-shower simulation uncertainties. The systematic uncertainty in the energy scale is estimated to be $14 \%$ (Verzi 2013).

Events above $20 \mathrm{EeV}$ recorded between 2004 January 1 and 2017 April 30 are used in this analysis. Above $20 \mathrm{EeV}$, both "vertical showers" (zenith angle $\theta<60^{\circ}$; Abraham et al. 2010) and "inclined showers" $\left(60^{\circ} \leqslant \theta \leqslant 80^{\circ}\right.$; Aab et al. 2014b) trigger the array of detectors with $100 \%$ efficiency, the average angular resolution being below $1^{\circ}$ and the statistical energy resolution being better than $12 \%$.

Combining the vertical and inclined data sets, including unfolding correction factors as done in Aab et al. (2015a), enables sky coverage over the decl. range $-90^{\circ}<\delta<+45^{\circ}$. Using the same selection criteria as in Aab et al. (2015b), the total exposure for the period considered here is $89,720 \mathrm{~km}^{2}$ sr yr.

\section{Source Selection and UHECR Sky Models}

\subsection{Extragalactic Gamma-Ray Populations}

We extract our list of gamma-ray AGNs ( $\gamma$ AGNs hereafter) from the 2FHL catalog (Ackermann et al. 2016), which includes 360 sources detected by Fermi-LAT above $50 \mathrm{GeV}$. We study radio-loud objects within a $250 \mathrm{Mpc}$ radius, yielding 17 blazars and radio galaxies. Their $50 \mathrm{GeV}-2 \mathrm{TeV}$ integral flux is used as a proxy for the UHECR flux. Given the distance of these objects, the gamma-ray absorption by the extragalactic background light (e.g., Domínguez et al. 2011) is small.

The detections of seven SBGs have been reported using Fermi-LAT data: NGC 253, M82, NGC 4945, NGC 1068 (Ackermann et al. 2012), NGC 2146 (Tang et al. 2014), Arp 220 (Peng et al. 2016), and Circinus (Hayashida et al. 2013). Their gamma-ray luminosity has been shown to scale almost linearly with their continuum radio flux (Ackermann et al. 2012). We thus adopt as a proxy for the UHECR flux of SBGs their continuum emission at $1.4 \mathrm{GHz}$ for which a larger census exists.
We select the 23 SBGs with a flux larger than $0.3 \mathrm{Jy}$ among the 63 objects within $250 \mathrm{Mpc}$ searched for gamma-ray emission by Ackermann et al. (2012). Due to the possible incompleteness of that list near the Galactic plane $\left(|b|<10^{\circ}\right)$ and in the southern sky $\left(\delta<-35^{\circ}\right)$, relevant SBGs could be missing from our selection. However, we checked that our conclusions remain unchanged: ${ }^{100}$ (a) using all 63 objects listed in Ackermann et al. (2012), (b) using the catalog from Becker et al. (2009) with 32 SBGs above $0.3 \mathrm{Jy}$, (c) adding the Circinus SBG absent from (a) and (b), (d) using only the six SBGs reported in the 3FGL (NGC 253, M82, NGC 4945, NGC 1068, Circinus, NGC 2146; Acero et al. 2015) and their 1-100 GeV integral flux as a UHECR proxy.

\subsection{X-Ray and Infrared Samples}

Following previous searches (Aab et al. 2015b), we additionally study two flux-limited samples: Swift-BAT sources up to $250 \mathrm{Mpc}$, above a flux of $13.4 \times 10^{-12} \mathrm{erg} \mathrm{cm}^{-2} \mathrm{~s}^{-1}$, and sources from the 2MASS redshift survey (2MRS catalog; Huchra et al. 2012) beyond $1 \mathrm{Mpc}$, effectively taking out the Local Group as in Erdoğdu et al. (2006). We use the 14-195 keV flux and K-band flux corrected for Galactic extinction as UHECR proxies for each of these surveys.

The X-ray sky observed by Swift-BAT is dominated in flux by the nearby Centaurus A, often considered as a prime UHECR source candidate (e.g., Romero et al. 1996; Wykes et al. 2017), with additional diffuse structures arising from both radio-loud and radio-quiet AGNs. This constitutes a different selection of AGNs from that performed for the $\gamma$-ray sample (radio-loud only), dominated by the radio galaxies Centaurus A and M87 within $20 \mathrm{Mpc}$ and by the blazars Mrk 421 and Mrk 501 within $200 \mathrm{Mpc}$.

The 2MRS infrared intensity traces the distribution of extragalactic matter, and includes both star-forming galaxies and AGNs. It is dominated by contributions from the nearby SBG NGC 253, close to the South Galactic pole, M82, only observable from the northern hemisphere, along with M83 and NGC 4945, belonging to the same group of galaxies as Centaurus A. Strong emission from Centaurus A, as well as cumulated emission from fainter objects, e.g., in the Virgo cluster, constitute distinctive features of the 2MRS sky model with respect to the SBG one.

\subsection{Impact of Attenuation}

We account for attenuation of UHECRs from distant objects (GZK effect) using a data-driven scenario that reproduces the composition and spectral constraints obtained by the Observatory (Aab et al. 2017b). Assuming a homogeneous distribution of sources in the local universe, it was shown that an interpretation of the air-shower data using the EPOS-LHC interaction model together with a hard injection index $\gamma=1$ at the sources (scenario A) best matches the data, accounting for propagation effects (Aloisio et al. 2012; Alves Batista et al. 2016). We also consider two other scenarios matching the data reasonably well: EPOSLHC with $\gamma=2$ (B) and Sibyll 2.1 with $\gamma=-1.5$ (C). These scenarios differ in the composition and maximum rigidities attainable at the sources. For each scenario and a chosen energy threshold, we evaluate the flux attenuation factor due

\footnotetext{
${ }^{100}$ Test statistic for anisotropy within \pm 1 unit.
} 
Table 1

Populations Investigated

\begin{tabular}{|c|c|c|c|c|c|c|}
\hline SBGs & $l\left({ }^{\circ}\right)$ & $b\left(^{\circ}\right)$ & Distance $^{\mathrm{a}}(\mathrm{Mpc})$ & Flux Weight (\%) & Attenuated Weight: A/B/C (\%) & $\%$ Contribution $^{\mathrm{b}}: \mathrm{A} / \mathrm{B} / \mathrm{C}(\%)$ \\
\hline NGC 253 & 97.4 & -88 & 2.7 & 13.6 & $20.7 / 18.0 / 16.6$ & $35.9 / 32.2 / 30.2$ \\
\hline M82 & 141.4 & 40.6 & 3.6 & 18.6 & $24.0 / 22.3 / 21.4$ & $0.2 / 0.1 / 0.1$ \\
\hline NGC 4945 & 305.3 & 13.3 & 4 & 16 & $19.2 / 18.3 / 17.9$ & $39.0 / 38.4 / 38.3$ \\
\hline M83 & 314.6 & 32 & 4 & 6.3 & 7.6/7.2/7.1 & $13.1 / 12.9 / 12.9$ \\
\hline IC 342 & 138.2 & 10.6 & 4 & 5.5 & $6.6 / 6.3 / 6.1$ & $0.1 / 0.0 / 0.0$ \\
\hline NGC 6946 & 95.7 & 11.7 & 5.9 & 3.4 & $3.2 / 3.3 / 3.5$ & $0.1 / 0.1 / 0.1$ \\
\hline NGC 2903 & 208.7 & 44.5 & 6.6 & 1.1 & $0.9 / 1.0 / 1.1$ & $0.6 / 0.7 / 0.7$ \\
\hline NGC 5055 & 106 & 74.3 & 7.8 & 0.9 & $0.7 / 0.8 / 0.9$ & $0.2 / 0.2 / 0.2$ \\
\hline NGC 3628 & 240.9 & 64.8 & 8.1 & 1.3 & $1.0 / 1.1 / 1.2$ & $0.8 / 0.9 / 1.1$ \\
\hline NGC 3627 & 242 & 64.4 & 8.1 & 1.1 & $0.8 / 0.9 / 1.1$ & $0.7 / 0.8 / 0.9$ \\
\hline NGC 4631 & 142.8 & 84.2 & 8.7 & 2.9 & $2.1 / 2.4 / 2.7$ & $0.8 / 0.9 / 1.1$ \\
\hline M51 & 104.9 & 68.6 & 10.3 & 3.6 & $2.3 / 2.8 / 3.3$ & $0.3 / 0.4 / 0.5$ \\
\hline NGC 891 & 140.4 & -17.4 & 11 & 1.7 & $1.1 / 1.3 / 1.5$ & $0.2 / 0.3 / 0.3$ \\
\hline NGC 3556 & 148.3 & 56.3 & 11.4 & 0.7 & $0.4 / 0.6 / 0.6$ & $0.0 / 0.0 / 0.0$ \\
\hline NGC 660 & 141.6 & -47.4 & 15 & 0.9 & $0.5 / 0.6 / 0.8$ & $0.4 / 0.5 / 0.6$ \\
\hline NGC 2146 & 135.7 & 24.9 & 16.3 & 2.6 & $1.3 / 1.7 / 2.0$ & $0.0 / 0.0 / 0.0$ \\
\hline NGC 3079 & 157.8 & 48.4 & 17.4 & 2.1 & $1.0 / 1.4 / 1.5$ & $0.1 / 0.1 / 0.1$ \\
\hline NGC 1068 & 172.1 & -51.9 & 17.9 & 12.1 & $5.6 / 7.9 / 9.0$ & $6.4 / 9.4 / 10.9$ \\
\hline NGC 1365 & 238 & -54.6 & 22.3 & 1.3 & $0.5 / 0.8 / 0.8$ & $0.9 / 1.5 / 1.6$ \\
\hline Arp 299 & 141.9 & 55.4 & 46 & 1.6 & $0.4 / 0.7 / 0.6$ & $0.0 / 0.0 / 0.0$ \\
\hline Arp 220 & 36.6 & 53 & 80 & 0.8 & $0.1 / 0.3 / 0.2$ & $0.0 / 0.2 / 0.1$ \\
\hline NGC 6240 & 20.7 & 27.3 & 105 & 1 & $0.1 / 0.3 / 0.1$ & $0.1 / 0.3 / 0.1$ \\
\hline Mkn 231 & 121.6 & 60.2 & 183 & 0.8 & $0.0 / 0.1 / 0.0$ & $0.0 / 0.0 / 0.0$ \\
\hline \multicolumn{7}{|l|}{$\gamma \mathrm{AGNs}$} \\
\hline Cen A Core & 309.6 & 19.4 & 3.7 & 0.8 & $60.5 / 14.6 / 40.4$ & $86.8 / 56.3 / 71.5$ \\
\hline M87 & 283.7 & 74.5 & 18.5 & 1 & $15.3 / 7.1 / 29.5$ & $9.7 / 12.1 / 23.1$ \\
\hline NGC 1275 & 150.6 & -13.3 & 76 & 2.2 & $6.6 / 6.1 / 7.5$ & $0.7 / 1.6 / 1.0$ \\
\hline IC 310 & 150.2 & -13.7 & 83 & 1 & $2.3 / 2.4 / 2.6$ & $0.3 / 0.6 / 0.3$ \\
\hline $3 \mathrm{C} 264$ & 235.8 & 73 & 95 & 0.5 & $0.8 / 1.0 / 0.8$ & $0.4 / 1.3 / 0.5$ \\
\hline TXS $0149+710$ & 127.9 & 9 & 96 & 0.5 & $0.7 / 0.9 / 0.7$ & $0.0 / 0.0 / 0.0$ \\
\hline Mkn 421 & 179.8 & 65 & 136 & 54 & $11.4 / 48.3 / 14.7$ & $1.8 / 19.1 / 2.8$ \\
\hline PKS 0229-581 & 280.2 & -54.6 & 140 & 0.5 & $0.1 / 0.5 / 0.1$ & $0.2 / 2.0 / 0.3$ \\
\hline Mkn 501 & 63.6 & 38.9 & 148 & 20.8 & $2.3 / 15.0 / 3.6$ & $0.3 / 5.2 / 0.6$ \\
\hline $1 \mathrm{ES} 2344+514$ & 112.9 & -9.9 & 195 & 3.3 & $0.0 / 1.0 / 0.1$ & $0.0 / 0.0 / 0.0$ \\
\hline Mkn 180 & 131.9 & 45.6 & 199 & 1.9 & $0.0 / 0.5 / 0.0$ & $0.0 / 0.0 / 0.0$ \\
\hline $1 \mathrm{ES} 1959+650$ & 98 & 17.7 & 209 & 6.8 & $0.0 / 1.7 / 0.1$ & $0.0 / 0.0 / 0.0$ \\
\hline AP Librae & 340.7 & 27.6 & 213 & 1.7 & $0.0 / 0.4 / 0.0$ & $0.0 / 1.3 / 0.0$ \\
\hline TXS $0210+515$ & 135.8 & -9 & 218 & 0.9 & $0.0 / 0.2 / 0.0$ & $0.0 / 0.0 / 0.0$ \\
\hline GB6 J0601 + 5315 & 160 & 14.6 & 232 & 0.4 & $0.0 / 0.1 / 0.0$ & $0.0 / 0.0 / 0.0$ \\
\hline PKS 0625-35 & 243.4 & -20 & 245 & 1.3 & $0.0 / 0.1 / 0.0$ & $0.0 / 0.5 / 0.0$ \\
\hline I Zw 187 & 77.1 & 33.5 & 247 & 2.3 & $0.0 / 0.2 / 0.0$ & $0.0 / 0.0 / 0.0$ \\
\hline
\end{tabular}

Notes.

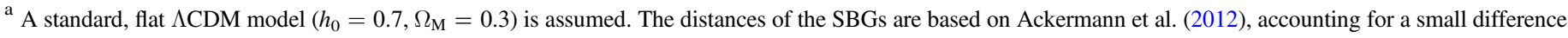
in $h_{0}$. The distances of the $\gamma \mathrm{AGNs}$ are based on their redshifts, except for the nearby Cen A (Tully et al. 2013).

$\mathrm{b} \%$ contributions account for the directional exposure of the array.

to propagation for each source and correct its expected UHECR flux accordingly.

The two extragalactic gamma-ray populations under study and the relative weight of each source are provided in Table 1. The relative contributions accounting for the directional exposure of the Observatory are shown in the last column. Because SBGs are mostly nearby, attenuation from them is much less important than that from the more distant blazars in the $\gamma \mathrm{AGN}$ sample. Taking into account attenuation, $\sim 90 \%$ of the accumulated flux from SBGs emerges from a $\sim 10 \mathrm{Mpc}$ radius region, while the radius goes up to $\sim 150 \mathrm{Mpc}$ for $\gamma$ AGNs. For both the 2MRS and Swift-BAT flux-limited samples, the $90 \%$ radius is $\sim 70 \mathrm{Mpc}$.

\section{Analysis and Results}

\subsection{Maximum-likelihood Analysis}

We build the UHECR sky model as the sum of an isotropic component plus the anisotropic contribution from the sources. For the anisotropic component, each source is modeled as a Fisher distribution (Fisher 1953), the equivalent of a Gaussian on the sphere. Its distribution is centered on the coordinates of 

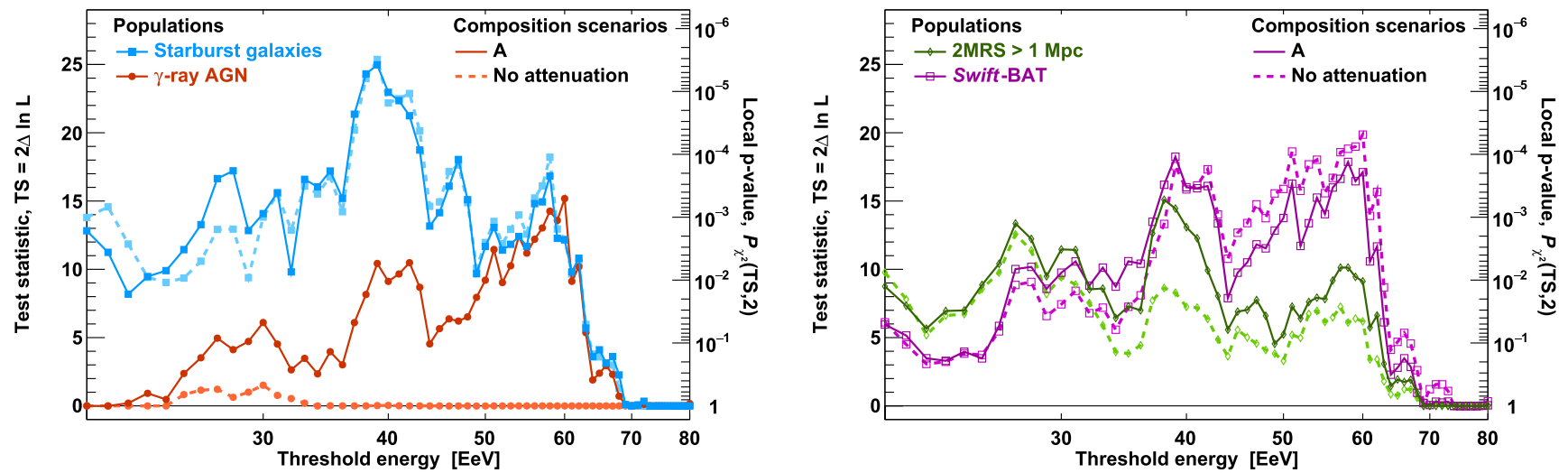

Figure 1. TS scan over the threshold energy for SBGs and AGNs (left) and Swift-BAT and 2MRS sources (right), including attenuation (lighter dashed lines) or not (darker solid lines).

the source, the integral being set by its flux attenuated above the chosen energy threshold, and the angular width—or search radius ${ }^{101}$ _ being a free parameter common to all sources. No shift of the centroid position is considered, avoiding dependence on any particular model of the Galactic magnetic field in this exploratory study. After mixing the anisotropic map with a variable fraction of isotropy, as in Abreu et al. (2010), the model map is multiplied by the directional exposure of the array and its integral is normalized to the number of events. The model map thus depends on two variables aimed at maximizing the degree of correlation with UHECR events: the fraction of all events due to the sources (anisotropic fraction) and the rms angular separation between an event and its source (search radius) in the anisotropic fraction.

We perform an unbinned maximum-likelihood analysis, where the likelihood (L) is the product over the UHECR events of the model density in the UHECR direction. The test statistic (TS) for deviation from isotropy is the likelihood ratio test between two nested hypotheses: the UHECR sky model and an isotropic model (null hypothesis). The TS is maximized as a function of two parameters: the search radius and the anisotropic fraction. We repeat the analysis for a sequence of energy thresholds.

For a given energy threshold, we confirmed with simulations that the TS for isotropy follows a $\chi^{2}$ distribution with two degrees of freedom, as expected (Wilks 1938), directly accounting for the fit of two parameters of the model. As in Aab et al. (2015b), we penalize the minimum $p$-value for a scan in threshold energy, by steps of $1 \mathrm{EeV}$ up to $80 \mathrm{EeV}$, estimating the penalty factor with Monte-Carlo simulations. The $p$-values are converted into significances assuming 1-sided Gaussian distributions.

\subsection{Single Population against Isotropy}

Previous anisotropy studies (e.g., Aab et al. 2015b) have considered a scan in energy threshold starting at $40 \mathrm{EeV}$, where the observed flux reaches half the value expected from lowerenergy extrapolations, but as shown in Figure 1, there is a maximum in the significance close to this starting point. Therefore we have evaluated the TS down to $20 \mathrm{EeV}$.

The TS is maximum for SBGs above $39 \mathrm{EeV}$ (894 events), with or without attenuation. For $\gamma \mathrm{AGNs}$, the TS is maximum above $60 \mathrm{EeV}$ (177 events) after accounting for attenuation. As shown in Figure 1, left, attenuation mildly impacts SBGs that are nearby: we obtain TS $=24.9 / 25.5 / 25.7$ for scenarios

\footnotetext{
101 Inverse square root of Fisher's concentration parameter.
}

A/B/C, respectively. The impact is more pronounced for $\gamma$ AGNs, a larger attenuation reducing contributions from distant blazars: we obtain a maximum TS of 15.2/9.4/11.9 for scenarios A/B/C. Shifting the energy scale within systematic uncertainties $( \pm 14 \%)$ affects the maximum TS by \pm 1 unit for $\gamma$ AGNs, \pm 0.3 for SBGs.

Penalizing for the energy scan, the maximum TS obtained for SBGs and $\gamma \mathrm{AGNs}$ within scenario A corresponds to $4.0 \sigma$ and $2.7 \sigma$ deviations from isotropy, respectively. As shown in Figure 2 (left), the maximum deviation for $\gamma \mathrm{AGNs}$ is found at an angular scale of $7_{-2}^{+4 \circ}$ and a $7 \pm 4 \%$ fraction of anisotropic events. For SBGs, a stronger deviation from isotropy is uncovered at an intermediate angular scale of $13_{-3}^{+4}$ and an anisotropic fraction of $10 \pm 4 \%$. The systematic uncertainty induced by the energy scale and attenuation scenario is at the level of $0.3 \%$ for the anisotropic fraction and 0.5 for the search radius obtained with SBGs.

For Swift-BAT and 2MRS sources attenuated within scenario A, we obtain maximum TSs of $18.2(3.2 \sigma)$ above $39 \mathrm{EeV}$ and $15.1(2.7 \sigma)$ above $38 \mathrm{EeV}$, respectively (see Figure 1, right). These correspond to values of the best-fit parameters of $12_{-4}^{+60}$ and $7_{-3}^{+4} \%$ for Swift-BAT, $13_{-4}^{+7 \circ}$ and $16_{-7}^{+8} \%$ for 2 MRS.

The different degrees of anisotropy obtained from each catalog can be understood from Figure 3 (top) showing a UHECR hotspot in the direction of the Centaurus A/M83/ NGC 4945 group. The $\gamma$ AGN model $(>60 \mathrm{EeV})$ and SwiftBAT model $(>39 \mathrm{EeV})$ are dominated by Centaurus A, which is $7^{\circ}$ and $13^{\circ}$ away from NGC 4945 and M83, respectively. The starburst model additionally captures the UHECR excess close to the Galactic South Pole, interpreted as contributions from NGC 1068 and NGC 253, yielding an increase in the anisotropy signal from $\sim 3 \sigma$ to $4 \sigma$. Additional diffuse contributions from clustered sources in the 2MRS catalog are not favored by the data, resulting in the smaller deviation from isotropy.

\subsection{Composite Models against Single Populations}

To compare the two distinct gamma-ray populations above their respective preferred thresholds, we investigate a composite model combining contributions from $\gamma$ AGNs and SBGs, adopting a single search radius and leaving the fraction of events from each population free. The TS in this case is the difference between the maximum likelihood of the combined model and that of the null hypothesis of a single population at 
Starburst galaxies - E > $39 \mathrm{EeV}$

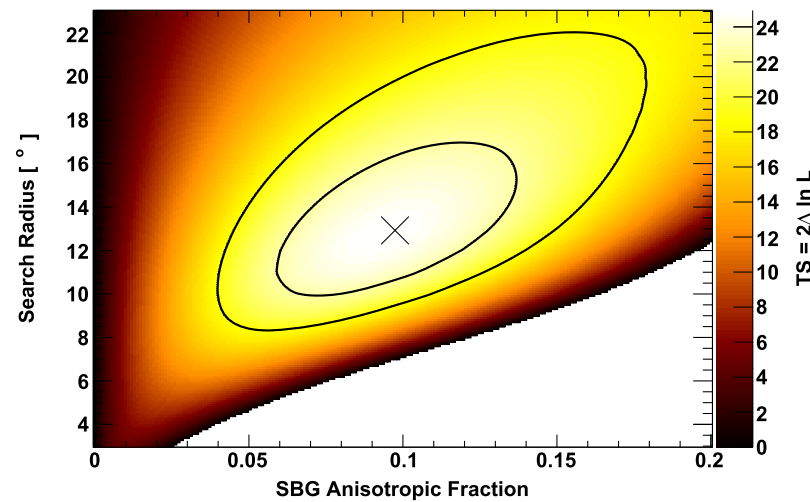

Active galactic nuclei - $\mathrm{E}>60 \mathrm{EeV}$

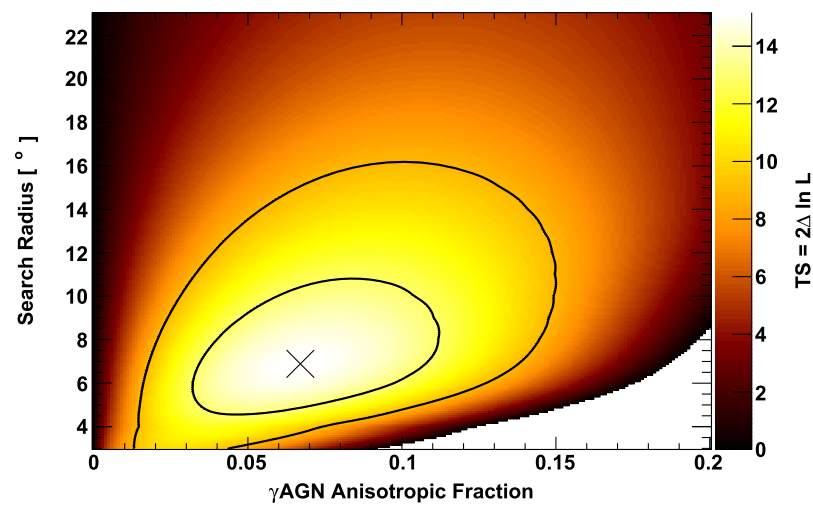

SBG and $\gamma$ AGN - E > $39 \mathrm{EeV}$

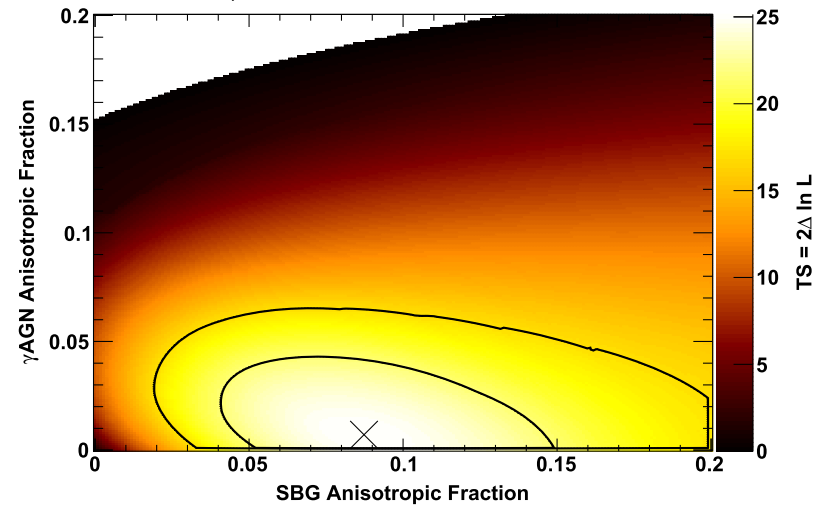

SBG and $\gamma$ AGN - E > $60 \mathrm{EeV}$

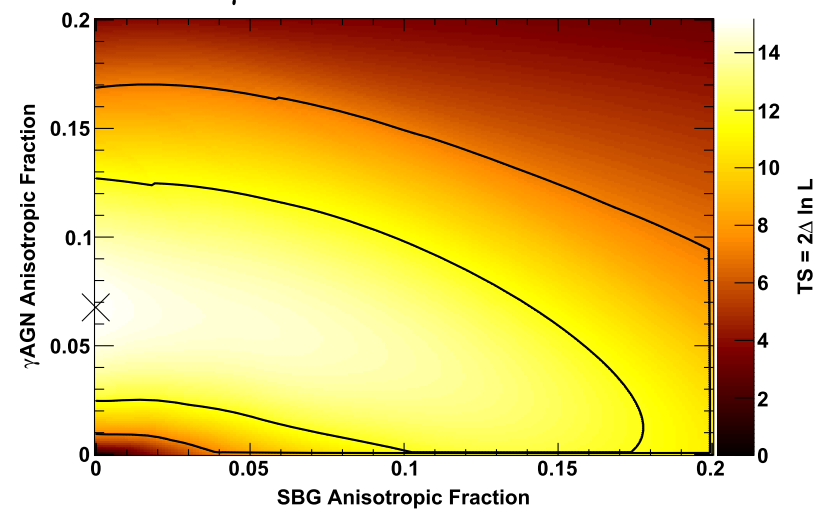

Figure 2. TS profile above $39 \mathrm{EeV}$ (top) and $60 \mathrm{EeV}$ (bottom) over the fit parameters for SBG-only and $\gamma \mathrm{AGN}$-only models (left) and for composite models including both SBGs and $\gamma$ AGNs with the same free search radius (right). The lines indicate the $1 \sigma-2 \sigma$ regions.

Table 2

Results-Scenario A

\begin{tabular}{|c|c|c|c|c|c|c|c|c|c|}
\hline $\begin{array}{l}\text { Test } \\
\text { Hypothesis }\end{array}$ & $\begin{array}{l}\text { Null } \\
\text { Hypothesis }\end{array}$ & $\begin{array}{l}\text { Threshold } \\
\text { Energy }^{\mathrm{a}}\end{array}$ & TS & $\begin{array}{c}\text { Local } p \text {-value } \\
\left.\mathcal{P}_{\chi^{2}} \text { (TS, } 2\right)\end{array}$ & $\begin{array}{l}\text { Post-trial } \\
p \text {-value }\end{array}$ & $\begin{array}{c}\text { 1-sided } \\
\text { Significance }\end{array}$ & $\begin{array}{l}\text { AGN/Other } \\
\text { Fraction }\end{array}$ & $\begin{array}{c}\text { SBG } \\
\text { Fraction }\end{array}$ & $\begin{array}{l}\text { Search } \\
\text { Radius }\end{array}$ \\
\hline SBG + ISO & ISO & $39 \mathrm{EeV}$ & 24.9 & $3.8 \times 10^{-6}$ & $3.6 \times 10^{-5}$ & $4.0 \sigma$ & $\mathrm{N} / \mathrm{A}$ & $9.7 \%$ & $12: 9$ \\
\hline$\gamma \mathrm{AGN}+\mathrm{SBG}+\mathrm{ISO}$ & $\gamma \mathrm{AGN}+\mathrm{ISO}$ & $39 \mathrm{EeV}$ & 14.7 & $\mathrm{~N} / \mathrm{A}$ & $1.3 \times 10^{-4}$ & $3.7 \sigma$ & $0.7 \%$ & $8.7 \%$ & 12.5 \\
\hline$\gamma \mathrm{AGN}+\mathrm{ISO}$ & ISO & $60 \mathrm{EeV}$ & 15.2 & $5.1 \times 10^{-4}$ & $3.1 \times 10^{-3}$ & $2.7 \sigma$ & $6.7 \%$ & N/A & 6.9 \\
\hline$\gamma \mathrm{AGN}+\mathrm{SBG}+\mathrm{ISO}$ & $\mathrm{SBG}+\mathrm{ISO}$ & $60 \mathrm{EeV}$ & 3.0 & $\mathrm{~N} / \mathrm{A}$ & 0.08 & $1.4 \sigma$ & $6.8 \%$ & $0.0 \%^{\mathrm{b}}$ & $7: 0$ \\
\hline Swift-BAT + ISO & ISO & $39 \mathrm{EeV}$ & 18.2 & $1.1 \times 10^{-4}$ & $8.0 \times 10^{-4}$ & $3.2 \sigma$ & $6.9 \%$ & $\mathrm{~N} / \mathrm{A}$ & 12.3 \\
\hline 2MRS + ISO & ISO & $38 \mathrm{EeV}$ & 15.1 & $5.2 \times 10^{-4}$ & $3.3 \times 10^{-3}$ & $2.7 \sigma$ & $15.8 \%$ & $\mathrm{~N} / \mathrm{A}$ & $13: 2$ \\
\hline $2 \mathrm{MRS}+\mathrm{SBG}+\mathrm{ISO}$ & $2 \mathrm{MRS}+\mathrm{ISO}$ & $39 \mathrm{EeV}$ & 10.4 & $\mathrm{~N} / \mathrm{A}$ & $1.3 \times 10^{-3}$ & $3.0 \sigma$ & $1.1 \%$ & $8.9 \%$ & 12.6 \\
\hline
\end{tabular}

Notes. ISO: isotropic model.

${ }^{a}$ For composite model studies, no scan over the threshold energy is performed.

${ }^{\mathrm{b}}$ Maximum TS reached at the boundary of the parameter space.

the selected energy threshold. The parameter added to the more complex model results in a $\chi^{2}$ distribution with one degree of freedom.

The best-fit anisotropic fractions obtained for the composite model (free search radius) are shown in Figure 2 (right). Above $39 \mathrm{EeV}$, the $\gamma \mathrm{AGN}$-only model is disfavored by $3.7 \sigma$ relative to a combined model with a 9\% contribution from SBGs and $1 \%$ contribution from $\gamma$ AGNs. Above $60 \mathrm{EeV}$, the TS obtained with the composite model is not significantly higher than what is obtained by either model. This is illustrated in Figure 2 (right) by the agreement at the $1 \sigma$ level of a model including $0 \%$ SBGs $/ 7 \% \gamma$ AGNs with a model including $13 \%$ SBGs $/ 0 \%$ $\gamma$ AGNs above $60 \mathrm{EeV}$.

As summarized in Table 2, composite models including SBGs and either 2MRS or Swift-BAT sources best match the data above $39 \mathrm{EeV}$ for $9 \%-7 \%$ fractions of events associated to 


\section{Observed Excess Map - E > 39 Eev}

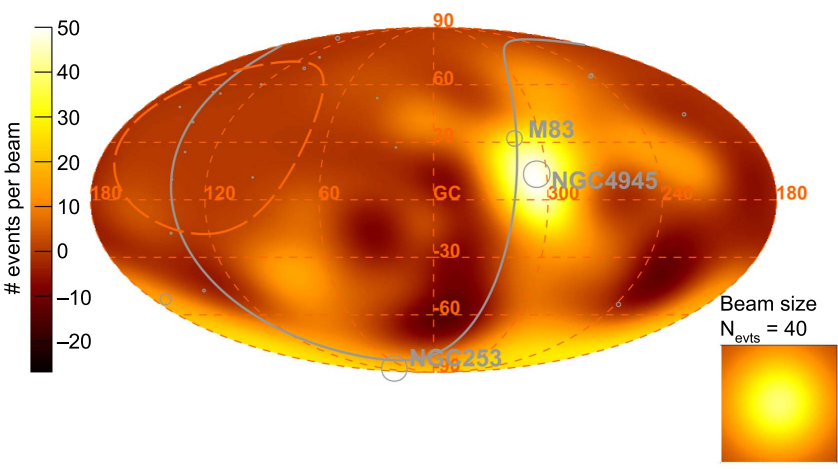

Model Excess Map - Starburst galaxies - E > $39 \mathrm{EeV}$

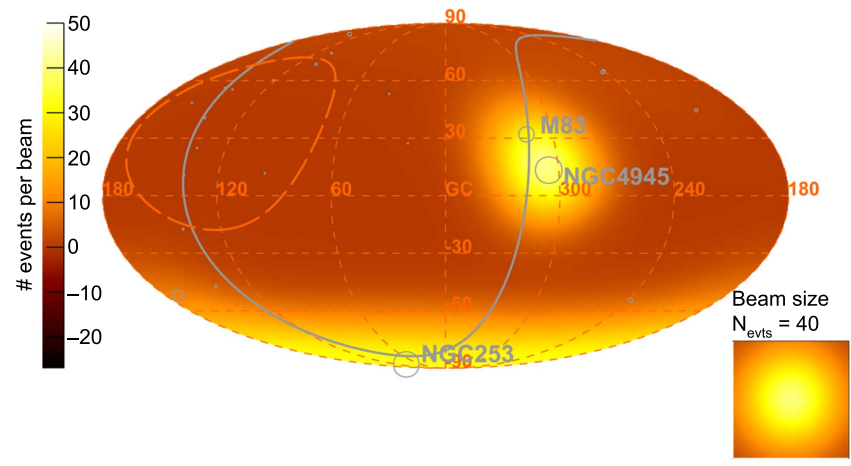

Residual Excess Map - Starburst galaxies - E > $39 \mathrm{EeV}$

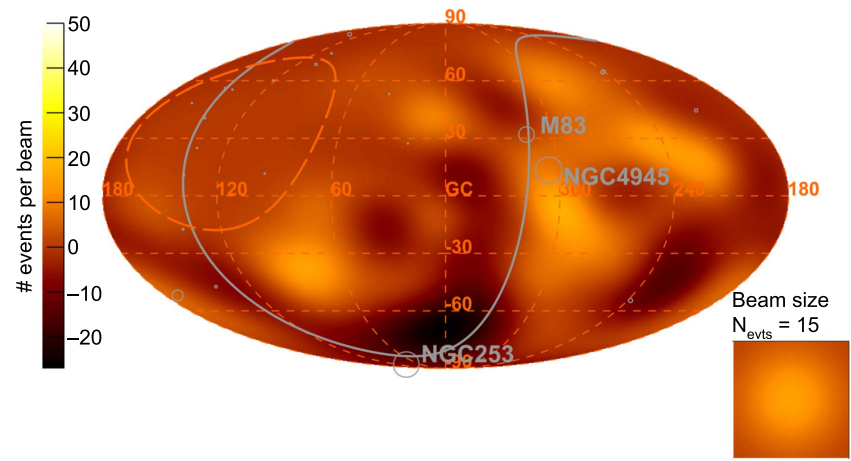

Model Flux Map - Starburst galaxies - E > $39 \mathrm{EeV}$

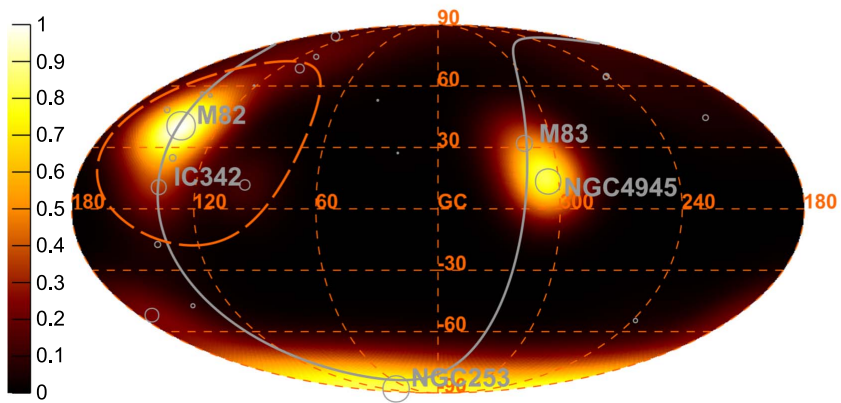

Observed Excess Map - E > 60 Eev

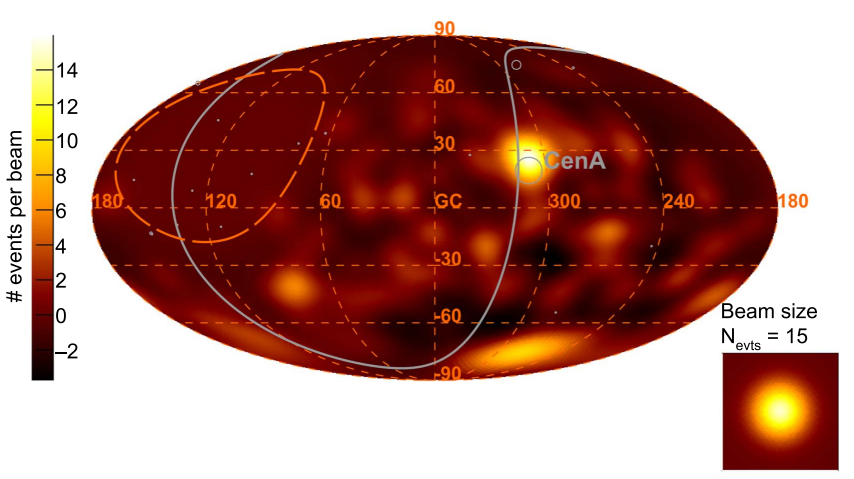

Model Excess Map - Active galactic nuclei - E > $60 \mathrm{EeV}$

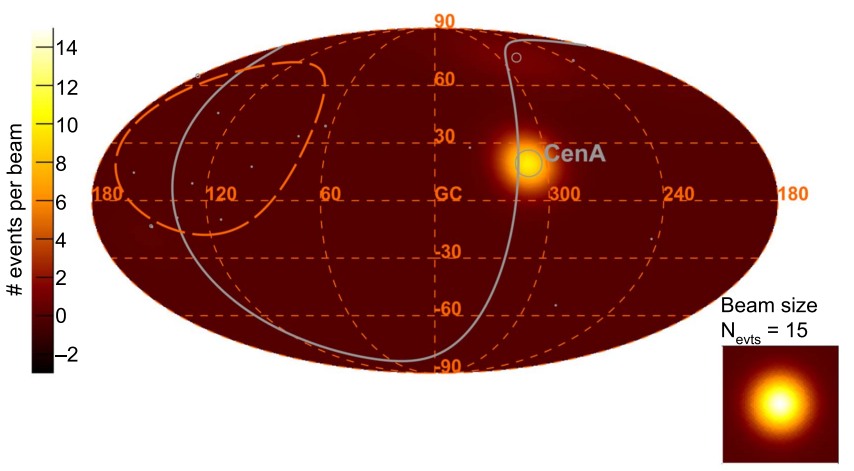

Residual Excess Map - Active galactic nuclei - E > $60 \mathrm{EeV}$

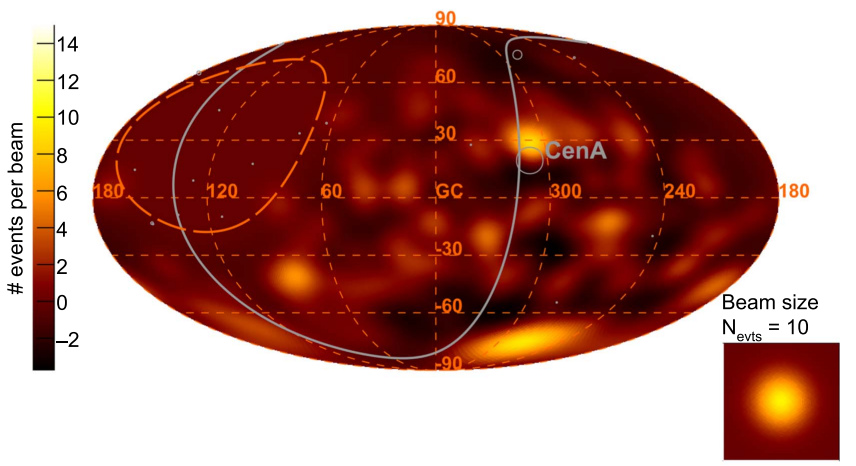

Model Flux Map - Active galactic nuclei - E > $39 \mathrm{EeV}$

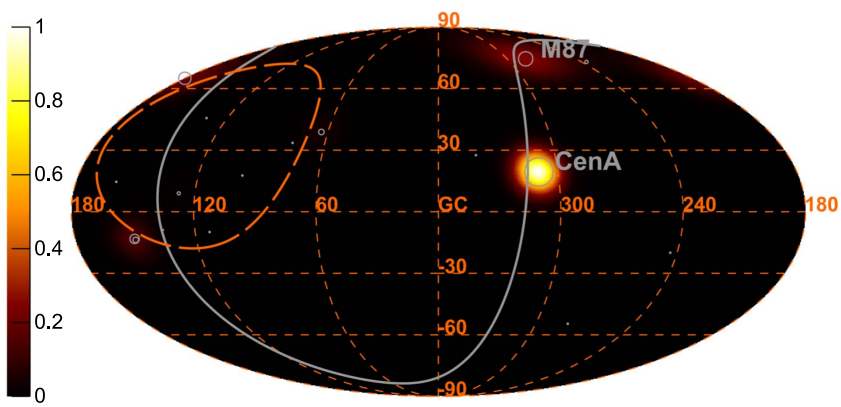

Figure 3. Top to bottom: observed excess map; model excess map; residual map; model flux map, for the best-fit parameters obtained with SBGs above 39 EeV (left) and $\gamma$ AGNs above $60 \mathrm{EeV}$ (right). The excess maps (best-fit isotropic component subtracted) and residual maps (observed minus model) are smeared at the best-fit angular scale. The color scale indicates the number of events per smearing beam (see inset). The model flux map corresponds to a uniform full-sky exposure. The supergalactic plane is shown as a solid gray line. An orange dashed line delimits the field of view of the array.

(FITS files for this figures are available.) 
SBGs and $1 \%-3 \%$ to the flux-limited samples. A $3.0 \sigma-2.6 \sigma$ advantage is found for the composite models including SBGs with respect to the 2MRS-only and Swift-BAT-only models.

\section{Discussion}

We have compared the arrival directions of UHECRs detected at the Pierre Auger Observatory with two distinct gamma-ray samples and two flux-limited samples of extragalactic sources. Our comparison with SBGs shows that isotropy of UHECRs is disfavored with $4.0 \sigma$ confidence, accounting for the two free parameters and including the penalty for scanning over energy thresholds. This is the most significant evidence found in this study for anisotropy of UHECRs on an intermediate angular scale. It should be noted, however, that numerous anisotropy studies have been conducted with data from the Observatory, not only those that have been published by the Collaboration. There is no rigorous way to evaluate a statistical penalty for other searches.

The pattern of UHECR arrival directions is best matched by a model in which about $10 \%$ of those cosmic rays arrive from directions that are clustered around the directions of bright, nearby SBGs. We evaluated the possibility of additional contributions from nearby $\gamma \mathrm{AGNs}$, such as Centaurus A, and from more distant sources through a comparison with samples tracing the distribution of extragalactic matter. We find that the contribution from SBGs to the indication of anisotropy is larger than that of the alternative catalogs tested. Nonetheless, caution is required in identifying SBGs as the preferred sources prior to understanding the impact of bulk magnetic deflections.

The sky maps used in this analysis are derived without incorporating any effects of the extragalactic or Galactic magnetic fields and winds (e.g., Pshirkov et al. 2011; Jansson \& Farrar 2012; Biermann et al. 2015). In particular, the arrival directions of UHECRs from a given source are modeled by a symmetric Fisher distribution centered on the source position. We checked the plausibility of the best-fit search radius obtained above $39 \mathrm{EeV}$ by simulating sky maps passed through the Galactic magnetic field from Jansson \& Farrar (2012), including a random component with a coherence length of $60 \mathrm{pc}$ as in Erdmann et al. (2016). For large deflections, UHECRs from a given SBG can leak in the direction of a neighboring source. The three composition scenarios discussed in Section 3.3 yield reconstructed search radii of $5^{\circ}-25^{\circ}$, bracketing the observed radius of $13^{\circ}$. The agreement is considered satisfactory given the uncertainties in our knowledge of the composition above $39 \mathrm{EeV}$ and of the deflections by the Galactic magnetic field (Unger \& Farrar 2017). Further studies aiming at possibly improving the model maps including deflections are underway.

It can be seen in Figure 3 (bottom) that M82 is expected to be one of the dominant sources in the full-sky starburst model presented here. Its declination of $\sim 70^{\circ} \mathrm{N}$ is outside the exposure of the Observatory but is covered in the northern Hemisphere by the Telescope Array (Abu-Zayyad et al. 2012). As noted, e.g., by Fang et al. (2014) and He et al. (2016), the excess of events observed at the Telescope Array (Abbasi et al. 2014) has some overlap with the position of M82, as well as with the position of the blazar Mkn 421 that would be a bright northern source in a low-attenuation scenario.

An analysis of full-sky data from the Pierre Auger Observatory and the Telescope Array may provide a more powerful test of the starburst and AGN models by probing all production regions of UHECRs. Combining the data is complicated, however, due to the spurious anisotropies that may be induced by possible mismatches in the relative exposures and/or systematic differences in the nominal energy scales used at each observatory. The first attempts at conducting such surveys are being made ( $\mathrm{Di}$ Matteo et al. 2017).

Additional exposure will bring better constraints on the brightest sources. At the same time, an instrumentation upgrade of the Observatory is being deployed on the water-Cherenkov detectors, adding a planar plastic scintillator of $4 \mathrm{~m}^{2}$ area to each of them (Aab et al. 2016b). The upgrade will provide mass-sensitive observables for each shower, enabling chargediscriminated studies with a duty cycle of nearly $100 \%$. Excluding highly charged nuclei from the analysis could eliminate a quasi-isotropic background that may mask the signature of individual sources imprinted by protons and other low-charge nuclei.

Finally, a large-scale dipolar anisotropy has been discovered above $8 \mathrm{EeV}$ (Aab et al. 2017a). While a direct connection between the large and intermediate angular-scale patterns has not yet been identified, the emergence of anisotropies at ultrahigh energy will certainly trigger further investigations of the scenarios underlying the production of UHECRs.

The successful installation, commissioning, and operation of the Pierre Auger Observatory would not have been possible without the strong commitment from the technical and administrative staff in Malargüe, and the financial support from a number of funding agencies in the participating countries, listed at https://www.auger.org/index.php/aboutus/funding-agencies.

\section{References}

Aab, A., Abreu, P., Aglietta, M., et al. 2014a, PhRvD, 90, 122006 Aab, A., Abreu, P., Aglietta, M., et al. 2014b, JCAP, 8, 019 Aab, A., Abreu, P., Aglietta, M., et al. 2015a, JCAP, 8, 049 Aab, A., Abreu, P., Aglietta, M., et al. 2015b, ApJ, 804, 15 Aab, A., Abreu, P., Aglietta, M., et al. 2015c, NIMPA, 798, 172 Aab, A., Abreu, P., Aglietta, M., et al. 2016a, PhLB, 762, 288 Aab, A., Abreu, P., Aglietta, M., et al. 2016b, arXiv:1604.03637 Aab, A., Abreu, P., Aglietta, M., et al. 2017a, Sci, 357, 1266 Aab, A., Abreu, P., Aglietta, M., et al. 2017b, JCAP, 4, 038 Abbasi, R. U., Abe, M., Abu-Zayyad, T., et al. 2014, ApJL, 790, L21 Abraham, J., Abreu, P., Aglietta, M., et al. 2010, NIMPA, 613, 29 Abreu, P., Aglietta, M., Ahn, E. J., et al. 2010, APh, 34, 314 Abu-Zayyad, T., Aida, R., Allen, M., et al. 2012, NIMPA, 689, 87 Acero, F., Ackermann, M., Ajello, M., et al. 2015, ApJS, 218, 23 Ackermann, M., Ajello, M., Allafort, A., et al. 2012, ApJ, 755, 164 Ackermann, M., Ajello, M., Atwood, W. B., et al. 2016, ApJS, 222, 5 Aloisio, R., Boncioli, D., Grillo, A. F., Petrera, S., \& Salamida, F. 2012, JCAP, 10,007

Alves Batista, R., Dundovic, A., Erdmann, M., et al. 2016, JCAP, 5, 038 Anchordoqui, L. A., Romero, G. E., \& Combi, J. A. 1999, PhRvD, 60, 103001 Becker, J. K., Biermann, P. L., Dreyer, J., \& Kneiske, T. M. 2009, arXiv:0901.1775

Biermann, P. L., Caramete, L. I., Fraschetti, F., et al. 2016, arXiv:1610.00944 Biermann, P. L., Caramete, L. I., Meli, A., et al. 2015, ASTRP, 2, 39

Dermer, C. D., \& Razzaque, S. 2010, ApJ, 724, 1366

Di Matteo, A., et al. 2017, JPSCP, 19, 011020

Domínguez, A., Primack, J. R., Rosario, D. J., et al. 2011, MNRAS, 410, 2556

Erdmann, M., Müller, G., Urban, M., \& Wirtz, M. 2016, APh, 85, 54

Erdoğdu, P., Huchra, J. P., Lahav, O., et al. 2006, MNRAS, 368, 1515

Fang, K., Fujii, T., Linden, T., \& Olinto, A. V. 2014, ApJ, 794, 126

Fisher, R. 1953, RSPSA, 217, 295 
Greisen, K. 1966, PhRvL, 16, 748

Hayashida, M., Stawarz, Ł., Cheung, C. C., et al. 2013, ApJ, 779, 131

He, H.-N., Kusenko, A., Nagataki, S., et al. 2016, PhRvD, 93, 043011

Hillas, A. M. 1984, ARA\&A, 22, 425

Huchra, J. P., Macri, L. M., Masters, K. L., et al. 2012, ApJS, 199, 26

Jansson, R., \& Farrar, G. R. 2012, ApJL, 761, L11

Peng, F.-K., Wang, X.-Y., Liu, R.-Y., Tang, Q.-W., \& Wang, J.-F. 2016, ApJL, 821, L20

Perley, D. A., Niino, Y., Tanvir, N. R., Vergani, S. D., \& Fynbo, J. P. U. 2016, SSRv, 202, 111

Pshirkov, M. S., Tinyakov, P. G., Kronberg, P. P., \& Newton-McGee, K. J. 2011, ApJ, 738, 192
Romero, G. E., Combi, J. A., Perez Bergliaffa, S. E., \& Anchordoqui, L. A. 1996, APh, 5, 279

Tang, Q.-W., Wang, X.-Y., \& Tam, P.-H. T. 2014, ApJ, 794, 26

Tully, R. B., Courtois, H. M., Dolphin, A. E., et al. 2013, AJ, 146, 86

Unger, M., \& Farrar, G. R. 2017, arXiv:1707.02339

Unger, M., Farrar, G. R., \& Anchordoqui, L. A. 2015, PhRvD, 92, 123001

Verzi, V. (for The Pierre Auger Collaboration) 2013, Proc. ICRC (Rio de Janeiro), 0928

Wilks, S. S. 1938, Ann. Math. Stat., 9, 60

Wykes, S., Taylor, A. M., Bray, J. D., Hardcastle, M. J., \& Hillas, A. M. 2017, arXiv: 1706.08229

Zatsepin, G. T., \& Kuz'min, V. A. 1966, JETPL, 4, 78 